\title{
Costos directos en el que incurre el Instituto Nacional de Salud del Niño en el tratamiento de niños quemados
}

\author{
Direct costs incurred in the National Institute of Child Health in the treatment of burned \\ children
}

Víctor Rodríguez ${ }^{1}$, Pablo Best-Bandenay ${ }^{2}$, Michelle Lozada-Urbano ${ }^{3,4}$

\section{RESUMEN}

Objetivo: Estimar los costos directos para el proveedor de los servicios asociados al accidente por quemaduras en la fase aguda, discriminar los costos de acuerdo a la extensión del área quemada; y edad del paciente. Métodos: Es una Investigación cuantitativa, descriptiva y de corte transversal. La población está conformada por el conjunto de historias clínicas (94) de los pacientes quemados atendidos en la unidad de quemados del Instituto Nacional de Salud del Niño. Las funciones de producción se definieron en 3 fases, para cada tipo de atención o tratamiento. Resultados: El tiempo promedio de hospitalización para los pequeños quemados según su extensión fue 19,47 días; para medianos quemados 28,97 días y para los quemados de gran extensión fue 39,48 días. Los niños fallecidos no se consideraron en el estudio. Las complicaciones más frecuentes fueron infecciones de las heridas e infecciones pulmonares. Resultado del Análisis de varianza (ANOVA) el valor $F, F=34,033 p<0,000$. Esta probabilidad es menor que el nivel de significancia usual del $5 \%(9,217 \mathrm{E}-12<0,05)$, indicando que hay diferencia significativa entre las medias de los tres grupos de quemados. El costo medio por paciente pequeño, mediano y grande quemado son (227 $97 ; 40385 ; 58934$ nuevos soles - US\$ 73.75; 130.65; 190.65 respectivamente). Los costos por autoinjerto es mayor en el grupo de quemados de gran extensión (3 30665 nuevos soles - US\$1070,2). Los costos por escarectomía van en aumento según la severidad del área de quemadura, pero no superan los costos del procedimiento autoinjerto. Conclusiones: La identificación y el análisis de los costos se consideran como un trazador de la problemática planteada alrededor de la discusión sobre la eficiencia de los sistemas de salud. Los resultados obtenidos servirán de insumo a la gestión de las autoridades de salud para el mejor diseño de programas de prevención y para una mejor toma de decisiones en cuanto a la secuencia del tratamiento y en la ciudadanía para una mayor sensibilización acerca del problema de las quemaduras.

Palabras clave: Costos económicos directos, unidad de quemados, costos directos.

\begin{abstract}
Objetives: Direct costs for the provider of the services associated with accident burns in the acute phase are estimated costs discriminate according to the extent of the burned area; and patient age. Methods: This is a crosssectional, descriptive and quantitative research. The population consists of the set of medical records (94) of burned patients treated at the burn unit of the National Institute of Child Health. Production functions defined in 3 stages for each type of care or treatment. Result: indicates that the average hospitalization time for small burned depending on their length was 19.47 days; 28.97 days for medium burned and burned large extension was 39.48 days. The dead children were not considered in the study. The most common complications were wound infections and lung infections. Result: Analysis of variance (ANOVA) F value, $F=34,033 p<0,000$. This probability is less than the usual level of significance of $5 \%(9,217 \mathrm{E}-12<0,05)$, indicating that no significant difference between the means of the three groups burned. The average cost per patient small, medium and large burned are $(227,97 ; 403,85 ; 589,34$ soles - US $\$ 73,75$; 130,$65 ; 190,65$ respectively). The costs are higher in the autograft group burned very long (3306.65 soles - US $\$$ 1,070.2). The costs are rising scarectomy according to the severity of the burn area, but not outweigh the costs of autograft procedure. Conclusions: The identification and analysis of costs are considered as a tracer of the issues raised about the discussion on the efficiency of health systems. The results serve as input to the management of health authorities to better design prevention programs and for better decision-making regarding the treatment sequence and citizens to improve awareness about the problem of burns.
\end{abstract}

Keywords: Direct economic costs, burn unit, direct costs

\footnotetext{
Instituto Nacional de Salud del Niño

Universidad Peruana Cayetano Heredia. Lima, Perú.

Universidad Peruana de Ciencias Aplicadas. Lima, Perú

Universidad Nacional José Faustino Sánchez Carrión.
} 


\section{INTRODUCCIÓN}

El aumento constante de los costos en el campo de los servicios de salud ha generado conciencia en el ámbito mundial sobre la necesidad de evaluar económicamente las decisiones médicas. (Rovira, 2004) En este sentido, hoy en día los profesionales de la salud deben tomar en consideración dentro de su actuación elementos de costo. En otras palabras, estamos frente a un nuevo pacto social entre el profesional de la salud y la sociedad, en el que habrá de considerar en sus acciones sanitarias las repercusiones económicas y sociales. (Jovell, 2005).

El accidente por quemaduras es la principal causa de mortalidad entre niños de 2 a 4 años en los pacientes del Instituto Nacional de Salud del Niño (INSN) Lima, Perú. No existen indicadores exactos de la cantidad de pacientes quemados sin embargo un estudio estadístico muestra que en el Perú hay una tasa de accidentabilidad de 3,9\% para la población menor de 15 años, lo cual significa que se tiene una gran cantidad de accidentes por quemaduras cerca del $75 \%$ son menores y se tratan en la casa, sin embargo el $23 \%$, son lo suficientemente extensas para requerir atención médica, y de ese porcentaje son suficientemente severas el $30 \%$ que van a requerir rehabilitación (INSN, 2008).

El consultorio externo del servicio de quemados en el INSN Breña en el año 2009, realizó 4675 atenciones $(1,6 \%$ del total de consultas externas); asimismo, se realizaron 703 cirugías en los pacientes quemados, se realizaron aproximadamente 5575 procedimientos de curación bajo anestesia general, asimismo se realizaron atenciones de rehabilitación y atenciones de psicología; durante ese mismo año, hubieron 304 egresos de la sala de hospitalización del servicio de Quemados (INSN, 2009).

No existen en la literatura estudios de los costos económicos generados por el tratamiento de los niños quemados en la fase aguda y en la etapa crónica o de secuela del accidente (INSN, 2008).

El objetivo fue estimar los costos directos para el proveedor de los servicios asociados al accidente por quemaduras en la fase aguda, discriminar los costos de acuerdo a la extensión del área quemada; y por la edad del paciente.

\section{MATERIAL Y MÉTODOS}

\section{Diseño del estudio}

El estudio es de diseño transversal, descriptivo $y$, de tipo cuantitativo. El análisis de costos es una forma parcial de evaluación económica aplicada en el ámbito sanitario. La evaluación parcial se da cuando sólo se examinan los costes de un proyecto, programa o servicio, pero comparando dentro del mismo dos o más alternativas de acción (orientadas hacia la consecución de un mismo objetivo), sin valorar de forma explícita ni la eficacia, ni la efectividad. Estas evaluaciones parciales, representan estadios intermedios vitales para la comprensión de los costes y los resultados de las alternativas consideradas (Rubio, 1995).

De acuerdo con Arredondo (1994), los estudios de costos para América Latina, deben efectuar una clasificación de costos distinta a las aplicadas en otros sistemas en base a lo siguiente: costo económico directo (para el sistema, y usuarios y familia), costo económico indirecto (implica la pérdida de productividad del paciente y familia durante la enfermedad) y los costos intangibles (dolor y sufrimiento a raíz de la enfermedad) En este sentido, el mismo autor, plantea una metodología de costeo por manejo de caso promedio o estándar, que inicia con el diseño de un instrumento de captación de la información, sigue con la identificación de insumos y funciones de producción para satisfacer la demanda hecha por expertos clínicos y de gestión (administrador, economista, epidemiólogo), se realiza la validación y ajuste con estudios de sombra (eficiente desde la perspectiva sanitaria y social), para, luego proceder a recopilar el costo por insumo o recurso. Su aplicación y procesamiento posterior de resultados permiten obtener los costos por función de producción (Arredondo, 1997).

Los recursos para mejorar la salud son escasos en relación a las necesidades, la priorización para asignación de los recursos debe hacerse necesariamente en base al criterio explícito. Un criterio válido es la eficacia o el maximizar el beneficio de los recursos disponibles. La relación entre los beneficios y el coste depende de la cantidad de actividad (Ortún, Pinto y Puig, 2001). En este sentido, el análisis marginal 
enfoca solamente en los costos extras o adicionales y, por tanto los beneficios de los cambios en el gasto de recursos. La eficacia global aumentará cuando la ganancia marginal en el beneficio excede la pérdida marginal. Como resultado, el análisis marginal identifica donde los recursos adicionales deben ser asignados, dónde deben hacerse las reducciones si el gasto debe recortarse, y cómo reasignarse los recursos para lograr una ganancia global en el beneficio sin el cambio global en el gasto para el servicio o institución (Cohen, 1994).

\section{Población y muestra}

El ámbito de estudio es el INSN. La población son los pacientes atendidos en el servicio de quemados En el año 2009 se atendieron un total de 356 pacientes. La muestra está conformada por 94 pacientes quemados atendidos durante el año 2009 en la unidad de quemados del INSN.

\section{Diseño muestral}

Para el cálculo del tamaño de la muestra, se utilizó la técnica estadística que corresponde a la fórmula que utiliza la varianza máxima de proporciones. El tamaño y la distribución para cada unidad muestral, considerando un nivel de confianza del $95,0 \%$, con un margen de error del $5 \%$ y con afijación proporcional al tipo de quemadura. Se ha estratificado a los pacientes quemados con el propósito de homogenizar el tipo de quemadura como: pequeña, mediana y grande. Se aplicó un muestreo aleatorio. La varianza de esta estimación se obtuvo combinando las varianzas de las estimaciones hechas en cada estrato. Como las variancias de cada estrato fueron pequeñas, dado que los estratos son relativamente homogéneos, la variancia final de la estimación combinada resultó también pequeña.

\section{Técnicas y procedimientos de recolección de datos}

Para estimar el costo de tratamiento en el que incurre la Unidad de Quemados del INSN Breña, se procede a la identificación del volumen de producción, las fases de producción, la identificación de la función de producción, la estimación de los recursos o factores de producción y la valoración de los mismos a través de la información brindada por diferentes instancias administrativas del establecimiento. Para el efecto de estimación de los costos económicos directos para el
INSN, es decir en aquellos que incurre sólo el hospital en la atención y recuperación del (a) paciente en el tratamiento de las quemaduras, se construyeron para cada caso la función de producción óptima desde una perspectiva técnica. Seguidamente, se recurrió a los protocolos o procedimientos establecidos por fase de atención y la estimación de tiempos y cantidades específicas de recursos o factores de producción.

Las funciones de producción se definieron en tres fases, para cada tipo de atención o tratamiento. Se iniciará el estudio, con la construcción de instrumentos de captación de información por etapa a partir de la identificación de la función de producción; para tal efecto se convocó un grupo interdisciplinario conformado por expertos clínicos en el tema de quemados; jefe de la unidad de costos hospitalarios, y miembros de las diferentes unidades del área de Administración, con la asesoría de un economista de la salud. Se consideraron como factores de producción: personal de salud, los medicamentos e insumos, los estudios de apoyo al diagnóstico, costos de capital (depreciación de equipos) y los gastos de hotelería. Seguidamente, se definirán los costos de hospitalización, procedimiento de curación, los costos equivalentes a las atenciones del psicólogo y terapista física, y se procederá a la valoración para la obtención del costo promedio. El periodo de referencia para la información sobre el costo de insumos fue el otorgado por la oficina de logística y costos para el año 2009. La información sobre recursos humanos, se logró a partir del tabulador de salarios de la institución, se determinó el costo/hora-hombre, considerando el cálculo del tiempo efectivo brindado al paciente por cada personal; para el caso de los medicamentos e insumos la base de cálculo fueron las cantidades promedio de uso y su costeo según la lista de precios provista por la unidad de logística. En el caso de los estudios de apoyo al diagnóstico (exámenes y procedimientos), se asumió el costo vigente (2009); y para los costos de capital, se valoraron en base a los equipos utilizados durante la atención, considerando los costos de los bienes y equipos facilitados por la Jefatura de logística, a partir de la depreciación de los mismos por medio del método de depreciación lineal. 
En el caso de los gastos de hotelería, se consideró: costos de capital (infraestructura) por área de hospitalización (depreciación); mobiliario clínico y, otros costos, entre ellos: Bienes, Servicios Públicos, Servicios generales, estos últimos se calcularon a partir de los costos promedios según los reportes de la unidad de costos hospitalarios por procedimientos y la estancia hospitalaria para cada caso.

Una vez validados y reajustados los instrumentos, se procederá a su aplicación utilizando las atenciones registradas en las historias clínicas facilitadas por archivo, se recopiló la información de los distintos rubros de costos de los recursos utilizados en las atenciones de la población estudiada. Seguidamente se utilizará la ficha de recopilación intermedia, donde se consolida por paciente los costos de cada recurso por fase y la ficha de recopilación final, que consigna los costos totales por paciente y por tipo de recurso. La valoración de los recursos se efectuará a través de la unidad de logística y de costos de la organización (a pesar de la limitación que ello presente).

\section{Consideraciones éticas}

El presente estudio puede ser catalogado exento de supervisión parcial o completa, al no trabajar directamente con pacientes. Asimismo, dado que la información es retrospectiva no se requiere consentimiento informado. La información recolectada se utilizará únicamente para fines de la investigación y, se respetaran las autorizaciones otorgadas por la unidad de quemados y el INSN.

\section{RESULTADOS}

En la Tabla 1. Se muestra el porcentaje de niños quemados por grupos de edad y según la extensión del área de quemados, se observa una mayor cantidad de niños en el grupo menor a dos años, siendo mayor en el grupo de los pequeños quemados.

Tabla 1. Porcentaje de niños por grupos de edad y según la extensión Del área de quemados, INSN.

\begin{tabular}{|c|c|c|c|c|c|c|c|c|}
\hline \multirow{3}{*}{ Edad de pacientes } & \multicolumn{6}{|c|}{ Extensión } & \multirow{2}{*}{\multicolumn{2}{|c|}{ TOTAL }} \\
\hline & \multicolumn{2}{|c|}{$\begin{array}{l}\text { Pequeños } \\
\text { quemados }\end{array}$} & \multicolumn{2}{|c|}{$\begin{array}{l}\text { Medianos } \\
\text { quemados }\end{array}$} & \multicolumn{2}{|c|}{$\begin{array}{c}\text { Grandes } \\
\text { quemados }\end{array}$} & & \\
\hline & $n$ & $\%$ & $n$ & $\%$ & $n$ & $\%$ & n & $\%$ \\
\hline Menores de 2 años & 14 & 143,75 & 14 & 40,00 & 9 & 33,33 & 37 & 39,36 \\
\hline Entre 2 y 6 años & 12 & 37,50 & 11 & 31,43 & 11 & 40,74 & 34 & 36,17 \\
\hline Mayores de 6 años & 6 & 18,75 & 10 & 28,57 & 7 & 25,93 & 23 & 24,47 \\
\hline TOTAL & 32 & 100,00 & 35 & 100,00 & 27 & 100,00 & 94 & 100,00 \\
\hline
\end{tabular}

Según la Tabla 2. El $69 \%$ de los niños quemados son de sexo masculino y el $31 \%$ corresponden a sexo femenino, siendo en los 3 grupos diferenciados por extensión del área de quemadura mayor el porcentaje de sexo masculino.

Tabla 2. Porcentaje de niños según la extensión del área quemada por sexo, INSN.

\begin{tabular}{|c|c|c|c|c|c|c|c|c|}
\hline \multirow{3}{*}{ Edad de pacientes } & \multicolumn{6}{|c|}{ Extensión } & \multirow{2}{*}{\multicolumn{2}{|c|}{ TOTAL }} \\
\hline & \multicolumn{2}{|c|}{$\begin{array}{l}\text { Pequeños } \\
\text { quemados }\end{array}$} & \multicolumn{2}{|c|}{$\begin{array}{l}\text { Medianos } \\
\text { quemados }\end{array}$} & \multicolumn{2}{|c|}{$\begin{array}{c}\text { Grandes } \\
\text { quemados }\end{array}$} & & \\
\hline & $n$ & $\%$ & $n$ & $\%$ & $n$ & $\%$ & n & $\%$ \\
\hline Masculino & 22 & 69 & 18 & 51 & 17 & 63 & 57 & 61 \\
\hline Femenino & 10 & 31 & 17 & 49 & 10 & 37 & 37 & 39 \\
\hline TOTAL & 32 & 100 & 35 & 100 & 27 & 100 & 94 & 100 \\
\hline
\end{tabular}


Tabla 3. Costos totales por actividades en el servicio de quemados, INSN.

\begin{tabular}{|c|c|c|c|}
\hline \multirow{3}{*}{$\begin{array}{c}\text { Actividades en el servicio } \\
\text { de quemados }\end{array}$} & \multicolumn{3}{|c|}{ Extensión } \\
\hline & $\begin{array}{l}\text { Pequeños } \\
\text { quemados }\end{array}$ & $\begin{array}{l}\text { Medianos } \\
\text { quemados }\end{array}$ & $\begin{array}{l}\text { Grandes } \\
\text { quemados }\end{array}$ \\
\hline & $\begin{array}{l}\text { promedios y } \\
\text { medianas }\end{array}$ & $\begin{array}{l}\text { promedios y } \\
\text { medianas }\end{array}$ & $\begin{array}{l}\text { promedios y } \\
\text { medianas }\end{array}$ \\
\hline \multirow[t]{2}{*}{ Días de hospitalización } & 19,47 & 28,97 & 39,48 \\
\hline & 17,50 & 28,00 & 28,00 \\
\hline \multirow[t]{2}{*}{ Total de costos por autoinjerto } & 641,41 & 1899,97 & 3306,65 \\
\hline & 05,00 & 1550,8 & 3149,6 \\
\hline \multirow[t]{2}{*}{ Costos por días de hospitalización } & 2742,90 & 4081,59 & 5562,00 \\
\hline & 2,47 & 3944,73 & 3944,73 \\
\hline \multirow{2}{*}{ Total de costos por escarectomía } & 570,65 & 979,90 & 2534,31 \\
\hline & 867,28 & 1164,00 & 225,90 \\
\hline \multirow[t]{2}{*}{ Total de costos por curaciones grandes } & & & 3222,75 \\
\hline & & & 2598,36 \\
\hline \multirow[t]{2}{*}{ Total de costos por curaciones medianas } & 1900,87 & 2954,29 & 1017,08 \\
\hline & 1712,40 & 2969,16 & 874,98 \\
\hline \multirow[t]{2}{*}{ Total de costos por curaciones pequeñas } & 526,52 & 878,45 & \\
\hline & 452,3 & 678,45 & \\
\hline \multirow{2}{*}{ Costos de medicamentos } & 615,50 & 1381,83 & 2021,75 \\
\hline & 535,1 & 1321,78 & 1423,75 \\
\hline \multirow{2}{*}{$\begin{array}{l}\text { Total de costos por atenciones en } \\
\text { psicología }\end{array}$} & & 49,40 & 66,4 \\
\hline & & 39,00 & 39,00 \\
\hline \multirow[t]{2}{*}{ Total de atenciones en terapia física } & & 86,54 & 132,88 \\
\hline & & 39,00 & 78,00 \\
\hline \multirow[t]{2}{*}{ Exámenes auxiliares } & 298,05 & 610,95 & 986,94 \\
\hline & 307,99 & 580,51 & 864,33 \\
\hline \multirow[t]{2}{*}{ COSTOS TOTALES1/ } & 7295,00 & 12923,33 & 18858,77 \\
\hline & 6697,59 & 12185,50 & 17894,00 \\
\hline
\end{tabular}

1/ Nuevos soles

La edad promedio según la extensión del área de quemadura es de 3,4 años para los quemados de pequeña extensión, y 4,4-4,7 años respectivamente para los quemados de mediana y gran extensión, Los días de hospitalización promedio, también van en aumento según la severidad del área de quemadura, para los quemados de pequeña extensión 19,47 para los de mediana extensión, 28,97 y para los de gran extensión 39,48 respectivamente ver Tabla 3.

Tabla 4. Descripción de los Costos Totales en la atención de pacientesen el servicio de quemados, INSN.

\begin{tabular}{|c|c|c|c|c|c|c|c|c|}
\hline \multirow[b]{2}{*}{$\begin{array}{l}\text { Extensión de } \\
\text { área de } \\
\text { quemadura }\end{array}$} & \multirow[b]{2}{*}{$\begin{array}{l}\text { Casos } \\
\text { totales }\end{array}$} & \multirow[b]{2}{*}{ Promedio } & \multirow[b]{2}{*}{$\begin{array}{c}\text { Desviación } \\
\text { típica }\end{array}$} & \multirow[b]{2}{*}{$\begin{array}{l}\text { Error } \\
\text { típico }\end{array}$} & \multicolumn{2}{|c|}{$95 \%$ Intervalo de confianza } & \multirow[b]{2}{*}{ Mínimo } & \multirow[b]{2}{*}{ Máximo } \\
\hline & & & & & $\begin{array}{l}\text { Límite } \\
\text { inferior }\end{array}$ & $\begin{array}{l}\text { Límite } \\
\text { superior }\end{array}$ & & \\
\hline 1 & 32 & 7295,87 & 3759,925 & 664,667 & 5940,27 & 8651,47 & 1997 & 14120 \\
\hline 2 & 35 & 12923,33 & 4874,697 & 823,974 & 11248,81 & 14597,84 & 4684 & 25532 \\
\hline 3 & 27 & 18850,77 & 7262,125 & 1397,597 & 15977,97 & 21723,57 & 7341 & 31416 \\
\hline Total $1 /$ & 94 & 12710,16 & 7013,907 & 723,429 & 11273,57 & 14146,75 & 1997 & 31416 \\
\hline
\end{tabular}

Fuente: Unidad de Estadística del INSN.

$1 /$ Nuevos soles 
En la Tabla 4, se describen los costos totales en la atención de pacientes. Los pacientes grandes quemados son más del doble que el costo promedio de los pequeños quemados.

Tabla 5. Resultado del ANOVA entre los costos totales por extensión del área de quemado, INSN.

\begin{tabular}{lrrrrr}
\hline & $\begin{array}{c}\text { Suma de } \\
\text { cuadrados }\end{array}$ & df & $\begin{array}{c}\text { Promedio de } \\
\text { cuadrados }\end{array}$ & F & Sig. \\
\hline Inter-grupos & $1,958 \mathrm{E}+09$ & 2 & $9,789 \mathrm{E}+08$ & 34,033 & $9,217 \mathrm{E}-12$ \\
Intra-grupos & $2,617 \mathrm{E}+09$ & 91 & $2,876 \mathrm{E}+07$ & \\
Total & $4,575 \mathrm{E}+09$ & 93 & & \\
\hline
\end{tabular}

En la Tabla 5. Resultado del ANOVA el valor $F$ (El valor $F$ corresponde a la división entre dos varianzas), $F=34,033 p<0,000$. Esta probabilidad es menor que el nivel de significancia usual del $5 \%(9,217 \mathrm{E}-12<0,05)$, indicando que hay diferencia significativa entre las medias de los tres grupos de quemados. Después de haber comprobado la diferencia significativa entre las medias, se comprueba el resultado de los contrastes a posteriori, (ver la Tabla 6) muestra con asterisco las parejas que son distintas. Se utilizó el método de contraste de Scheffé, que es más exigente que el de Tukey; es decir, exige una mayor diferencia entre las medias para encontrar resultados significativos.

Tabla 6. Comparación múltiple, estadísticos descriptivos por grupos, prueba de Scheffé.

\begin{tabular}{|c|c|c|c|c|c|c|}
\hline \multirow{2}{*}{ (I) Extensión } & \multirow{2}{*}{ (J) Extensión } & \multirow{2}{*}{$\begin{array}{l}\text { Diferencia de } \\
\text { medias (I-J) }\end{array}$} & \multirow{2}{*}{$\begin{array}{l}\text { Error } \\
\text { típico }\end{array}$} & \multirow{2}{*}{ Sig } & \multicolumn{2}{|c|}{ Intervalo de confianza al $95 \%$} \\
\hline & & & & & $\begin{array}{l}\text { Límite } \\
\text { inferior }\end{array}$ & $\begin{array}{l}\text { Límite } \\
\text { superior }\end{array}$ \\
\hline \multirow{2}{*}{1,00} & 2,00 & $-5627,456^{*}$ & 1311,720 &, 000 & $-8891,80$ & $-2363,12$ \\
\hline & 3,00 & $-11554,898^{*}$ & 1401,463 &, 000 & $-15042,57$ & $-8067,22$ \\
\hline \multirow{2}{*}{2,00} & 1,00 & $5627,456^{*}$ & 1311,720 &, 000 & 2363,12 & 8891,80 \\
\hline & 3,00 & $-5927,442^{*}$ & 1373,702 &, 000 & $-9346,03$ & $-2508,85$ \\
\hline \multirow{2}{*}{3,00} & 1,00 & $11554,898^{*}$ & 1401,463 &, 000 & 8067,22 & 15042,57 \\
\hline & 2,00 & $5927,442^{*}$ & 1373,702 &, 000 & $25,08,85$ & 9346,03 \\
\hline
\end{tabular}

${ }^{*}$. Es significativo al nivel 0.05 .

\section{DISCUSIÓN}

El tratamiento de pacientes hospitalizados por quemaduras puede tener costos asociados muy altos. Los recursos necesarios para proporcionar una atención óptima dada la cronicidad de la lesión de la quemadura son extensos. El impacto en los niños a veces de por vida también necesitará grandes recursos, para el sistema.

En este estudio hemos calculado el costo asociado desde la perspectiva del hospital y de los tratamientos de las lesiones. No es sorprendente que las variaciones del costo de Autoinjerto mostraran un comportamiento esperable en proporción directa con la extensión del área de los quemados, lo mismo sucedió con los costos por escarectomía. Los costos por Autoinjerto es mayor en el grupo de quemados de gran extensión (3306,65 nuevos soles - US $\$ 1,070)$. Los costos por Escarectomía van en aumento según la severidad del área de quemadura, pero no superan los costos del procedimiento denominado Autoinjerto.

En cuanto a los costos totales para todos los pacientes alcanzan 39077,1 nuevos soles US\$12,641. El costo total por paciente pequeño, mediano y grande quemado son (7295; 12923,33; 18858,77 nuevos soles - US\$ 2360; 4181; 6101 respectivamente), se hizo la conversión a dólares con precios a Marzo 2015. Esta gran diferencia se debió a los costos por días de hospitalización, a los costos por 
Escarectomía y los costos por autoinjerto que reciben los quemados de gran extensión.

Un estudio realizado en Valencia España encontró que el coste medio por paciente, incluyendo los costos sociales y laborales, fue $\$$ 95551 (dólares), con los costos de atención de salud por valor de sólo 10\% (Sánchez, Perepérez, Bastida y Martínez, 2007). En la India tuvieron un gasto total de US \$ 845,237 y costo por paciente en US \$1,060.5 (Ahuja y Goswami, 2013). Estudio desarrollado en Boston, USA describe el promedio del costo total de hospitalización de $\$ 83,535$ por paciente, en dólares del 2006 (Carey et al; 2012). Resultados en niños quemados de la ciudad de Seattle USA, han definido como costos totales a $\$ 9026$ dolares del 2005. Griffiths, et al; encontraron que la mayoría de las quemaduras de la infancia pueden ser tratados por 1,061 dólares por quemadura (Bass y Phillips, 2008). Estos valores reportados en diferentes estudios están muy por encima de los encontrados en esta investigación, muchos de los factores detallados como contribuyentes importantes a los costos hospitalarios son el estado pagador en la utilización de los recursos hospitalarios. La asociación entre nivel socioeconómico bajo y el resultado después de la lesión (Hass, 1994; Rhee, et al, 1997). Los pacientes con menor nivel socioeconómico tienden a tener peor acceso a atención en salud y peor estado de salud en general, lo que aumentaría susceptibilidad a las complicaciones de la lesión, como la como la infección que aumentaría la longitud del hospital de mantenerse y aumentar los costos. Además, los pacientes pediátricos de familias más pobres no pueden tener los recursos para proporcionar una atención adecuada en el hogar. Por lo tanto, los médicos pueden tener un mayor umbral de alta a los pacientes más pobres a sus hogares si es compleja las curaciones diarias que necesitan (DiGuiseppi, 2000).

El mayor porcentaje del costo directo de pacientes quemados corresponde a costos por hospitalización en pacientes quemados de gran extensión y en este mismo grupo a los costos por el tratamiento de Autoinjerto y costos de medicamentos.

El tiempo promedio de hospitalización para los pequeños quemados según su extensión fue
19,47 días; para medianos quemados 28,97 días y para los quemados de gran extensión fue 39,48 días. Estudios en la India encontraron que la media de la hospitalización para todas las admisiones fue 7,86 días (rango de 1 a 62 días) y para los sobrevivientes de 8,9 días (Ahuja y Goswami, 2013). Estudios en Boston USA, encontraron un promedio de 1,9 días (Carey et al; 2012). El tiempo de permanencia en el INSN, de los pacientes provenientes de la zona rural haría una tendencia a mantener estos pacientes en el hospital más tiempo, por tener peor acceso $\mathrm{y}$, hacer un seguimiento difícil.

Los totales de costos para las curaciones pequeñas y medianas se encuentran para los respectivos grupos por extensión de quemadura, sin embargo las curaciones grandes solo son para el grupo de quemados de gran extensión. Los totales correspondientes a medicamentos, son mayores para los quemados de gran extensión. Las derivaciones de los pacientes a psicología y a terapia física solo se dan para los grupos de pacientes quemados de mediana y gran extensión.

Las quemaduras siguen siendo una causa importante de morbilidad para los niños, con un impacto negativo en el desarrollo físico, mental y socioeconómico, y con un alto costo para la salud pública (Kai-Yang, 2009).

\section{Limitaciones}

El estudio se realizó en un establecimiento de primer nivel, donde el financiamiento es dado por el establecimiento. Por el diseño del estudio se tuvo en cuenta los costos directos, más no los costos indirectos, puesto que se requiere de mayor información difícil de cuantificar.

En cuanto a la muestra se hallaron costos extremos en los grupos homogéneos por extensión de quemadura, se vio por conveniente Normalizar la distribución quedándonos al final con una muestra de 94 pacientes.

\section{REFERENCIAS BIBLIOGRÁFICAS}

Ahuja, R.B. \& Goswami, P. (2013). Cost of providing inpatient burn care in a tertiary, teaching, hospital of North India. Burns, $2013 \mathrm{Jun}, 39$ ( 4 ), 558 64.doi:10.1016/j.burns.2013.01.013. 
Arredondo, A. (1994). Indicadores de eficiencia y los costos de producción en la atención médica. Revista de la Facultad de Ciencias Económicas de la Universidad Nacional Mayor San Marcos, 11, 20-21.

Arredondo, A. (1997). Costos económicos en la producción de servicios de salud: del costo de los insumos al costo de manejo de caso. Revista Salud Pública (México), 19,118-119

Bass, M.J. \& Phillips, L.G. (2008). Economics of pediatric burns. J Craniofac Surg $19(4): 888-90$ d o i : 10.1097/SCS.0b013e318175b58c.

Carey, K., Kazis, L.E., Lee, A.F., Liang, M.H., Li, N.C., Hinson, M.I., et al. (2012). Measuring the cost of care for children with acute burn injury. J Trauma Acute Care Surg. Sep;73(3 Suppl 2):S229-33. doi: 10.1097/TA.0b013e318265c88a.

Cohen, D. (1994). Marginal analysis in practice: an alternative to needs assessment for contracting health care. British Medical Journal (24 September), 309, 81-784.

DiGuiseppi, C. (2000). Roberts I. Individuallevel injury prevention strategies in the clinical setting. The future of children, $10,53-82$.

Haas, G. (1994). Acutely injured patients with trauma in Massachusetts: differences in care and mortality, by insurance status. Am J Public Health, 84, 1605 - 8.

Jovell, A. (2005). Contrato social y valores en la profesión médica. Revista de administración sanitaria siglo XXI, Vol. 3, (3), págs. 495-505.

Kai-Yang, L., Shi-Hui, Z., Hong-Tai, T., Yi-Tao, J., Zhao-Fan, X., Dao-Feng, B., et al. (2009). The direct hospitalization costs of pediatric scalds: 2-year results of a prospective case series. Burn Center, Changhai Hospital, Second Military Medical University, Shanghai 200433, China. Aug, 5(5), 738-45. Epub 2009 Mar 21.
Instituto Nacional de Salud del Niño (INSN). (2008). Informe Estadístico de la Unidad de Estadística

Instituto Nacional de Salud del Niño (INSN). (2009). Informe Estadístico de la Unidad de Estadística

Ortún, V., Pinto, L. \& Puig, J. (2001). La economía de la salud y su aplicación a la evaluación. Atención Primaria, 27 (1), 62-64.

Rubio, S. (1995). Glosario de Economía de la Salud. Madrid: Diaz de Santos.

Rhee, P., Grossman, D., Rivara, F., Mock, C., Jurkovich, G. \& Maier, R.V. (1997). The effect of payer status on utilization of hospital resources in trauma care. Arch Surg, 132:399-404.

Rovira, J. (2004). Evaluación económica en salud: de la investigación a la toma de decisiones. Rev. Esp. Salud Pública [online], vol.78, (3) [citado 2015-06-11], pp. 293-295. Disponible en: $<$ http://scielo.isciii.es/scielo.php?script= s c i arttext\&pid=S1135 $5727 \overline{2} 004000300001 \& \operatorname{lng}=e s \& n r m=i s o$ $>$. ISSN 1135-5727.

Sánchez, J.L., Perepérez, S.B., Bastida, J.L., \& Martínez, M.M. (2007). Cost-utility analysis applied to the treatment of burn patients in a specialized center. Arch Surg, 142(1), 50-7; discussion 57.

Correo electrónico: vraul27@gmail.com

Revisado por pares:

Recibido: 08-03-2015

Aprobado: 20-06-2015 